\title{
Automatic Vehicle Detection and Classification
}

\author{
Pedro M. Ferreira ${ }^{*}$, Gonçalo Marques ${ }^{*}$, Pedro M. Jorge ${ }^{*}$, Arnaldo J. Abrantes ${ }^{*}$ and António Amador ${ }^{+}$
}

\begin{abstract}
This paper presents a proposal for an automatic vehicle detection and classification (AVDC) system. The proposed AVDC should classify vehicles accordingly to the Portuguese legislation (vehicle height over the first axel and number of axels), and should also support profile based classification. The AVDC should also fulfill the needs of the Portuguese motorway operator, Brisa. For the classification based on the profile we propose the use of Eigenprofiles, a technique based on Principal Components Analysis. The system should also support multi-lane free flow for future integration in this kind of environments.
\end{abstract}

\section{INTRODUCTION}

$\mathrm{T}$ he goal of this work is to develop an automatic vehicle detection and classification (AVDC) system suited to the actual Portuguese legislation which classifies the vehicles based on the height of the vehicle at the first axel and the number of axels. In addition, this project is aiming to match the requirements of the multi-lane free flow (MLFF) environment and therefore should also support vehicle classification based on volumetric features (profile). This requirement can be achieved by creating a vertical laser curtain consisting of several basic AVDC units, like the one described in this paper, working in parallel.

The use of laser sensors for vehicle classification purposes is steadily increasing and companies like Efkon [2] and Kapsh [3] are already offering classification systems for single lane and multi-lane environments. Unfortunately, these commercial solutions are not suited to the Portuguese case as they are more directed to volumetric classification, derived from vehicle profile data acquired with a blade laser Sensor [4] or a LIDAR[5]. Furthermore, these technologies are seriously limited in speed due to the presence of mechanical parts. Nevertheless, if vehicles speed is not too fast, they obtain accurate $3 \mathrm{D}$ profiles and consequently high classification rates.

Other type of solution is to use a set of laser sensors to create a horizontal laser curtain [9] to produce reliable profiles. The main drawback of this kind of solutions is that they cannot cope with multi lane free flow environments.

There are also classification systems based on video features which replace the laser sensors by video cameras. The

* Those authors are with the Multimedia and Machine Learning Group, Department of Electronics, Telecommunications and Computer Engineering, ISEL, Lisbon. E-mail: pferreira@deetc.isel.ipl.pt, gmarques@deetc.isel.ipl.pt,_pmj@isel.ipl.pt and aja@isel.ipl.pt, respectively.

This author is with Brisa Auto-Estradas de Portugal, Lisbon. E-mail: Antonio.Amador@brisa.pt.

The project is sponsored by Brisa. diversity of solutions found in this type of systems is much higher as it is still an active research topic the choice of sensors and computer vision techniques [10-13].

As referred before, the goal of the AVDC described in this paper is to be able to classify accordingly to the Portuguese legislation. Moreover, the system should be able to classify vehicles based on their 2D profile (height vs. longitude). In this case, the speed of sensor laser is of mandatory importance as the profile accuracy depends on the number of measurements made over the first axel which has to be large even if the speed of the car is high.

In order to derive a volumetric classification system we propose, as first step, the use of Principal Components Analysis (PCA) to transform the 2D profile (raw data) into a set of uncorrelated features (Eigen Profiles) which will then be used as the input of the classifier. This approach has the advantage to be flexible, being easily adapted to other countries where this type of classification is used.

All the development of this project is taking under consideration the implementation of the AVDC in MLFF. Due to this goal, the AVDC could not be intrusive in the lane, and this consideration is one of the main reasons for the use of sensor lasers for measuring distances.

\section{Automatic Vehicle Detection AND ClAsSificAtion}

\section{A. Equipment}

The AVDC is being developed using a single pulse laser named Universal Laser Sensor (ULS) produced by Laser Technology Inc. [1]. The main advantages of this laser sensor are the high pulse rate and good accuracy. ULS has a pulse rate up to 4000 measures per second and $\pm 2 \mathrm{~cm}$ accuracy. This laser offers other important characteristics for this project, like the possibility of working in parallel by supporting an addressable protocol, the availability of a RS485 output which is ideal for long distances, and the absence of mechanical parts which leads to a longer longevity. The remaining features needed to perform the classification, namely axel detection and axel counting, they can be obtained by using a mechanical or fiber optic treadle [7] installed in the lane.

\section{B. Setup}

The AVDC setup consists on a ULS laser placed orthogonally to the pavement at the center of the lane. The ULS is responsible for the detection of the beginning and 
end of the vehicle. During this time interval, the AVDC collects all the measures sent by the ULS, creating the 2D profile and identifying the measures over the axels (see Fig. 1 to Figure 4). This way it is possible to get the 2D profile, the height over the first axel as well as the number of axels. The axels are detected using a treadle.

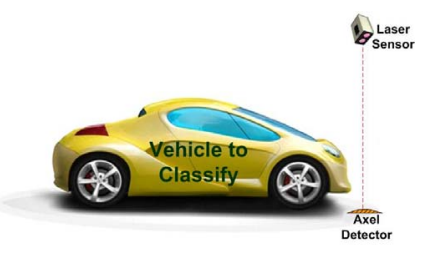

Fig. 1. Vehicle entering on the system.

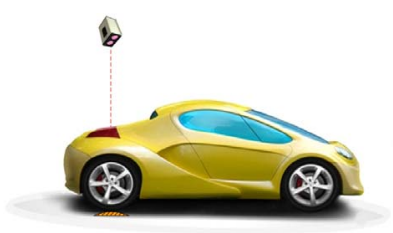

Fig. 3. Counting successive axels.

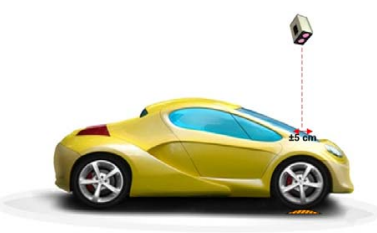

Fig. 2. Obtaining the height at the first axel and starting to count the number of axels.

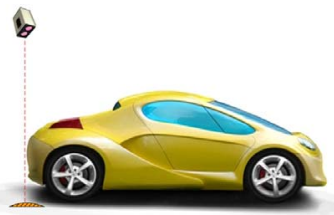

Fig. 4. Leaving the system after getting the signature (2D profile)

\section{System Requirements}

In order to guarantee the validity of the vehicle classification, the measure of the height at the first axel has to be inside a $\pm 5 \mathrm{~cm}$ interval around the first axel, with a precision of $\pm 2 \mathrm{~cm}$. The vehicle crosses the field of view of the system in a very short temporal window as it can be observed in Table 1. Therefore, the sensor sampling rate should be high enough (Table 2) to accommodate high vehicle speeds.

TABLE I

Speed vs. Duration (Considering vehicles with 4m of length).
\begin{tabular}{|c|c|c|}
\hline $\begin{array}{c}\text { Speed of the } \\
\text { Vehicle } \\
{[\mathrm{km} / \mathrm{h}]}\end{array}$ & $\begin{array}{c}\text { Temporal duration in the } \\
\text { system }\end{array}$ \\
\cline { 2 - 3 } & $\begin{array}{c}\text { Of the } \\
\text { Vehicle }[\mathrm{s}]\end{array}$ & $\begin{array}{c}\text { Of the first } \\
\text { Axel [ms] }\end{array}$ \\
\hline 25 & 0.576 & 14.4 \\
\hline 50 & 0.288 & 7.2 \\
\hline 75 & 0.192 & 4.8 \\
\hline 100 & 0.144 & 3.6 \\
\hline 120 & 0.120 & 3.0 \\
\hline 150 & 0.096 & 2.4 \\
\hline 200 & 0.072 & 1.8 \\
\hline
\end{tabular}

Other key aspect of the project is concerning to the synchronization between the system and both sensors. For the Portuguese classification system, this synchronization has to be particularly precise, since we need to know which measurement corresponds to the first axel.

A large amount of data is generated and processed for each car entering the system (2D profile) and therefore the overall system speed is a crucial design requirement as the classification task has to be performed in real time.
The speed and synchronization of the system become even more important when the system is used in MLFF. In this case, the number of laser sensors and axel detectors can be very large and the system should still be able to manage the different streams of the AVDC data, keeping its spatial and temporal coherence (for example, avoiding multiple classifications for the same car).

Several solutions can be devised for this problem, in the context of MLFF, and Figure 5 shows one such possible configuration for this implementation.

TABLE II

Sampling Rate vs. Speed.

\begin{tabular}{|c|c|c|}
\multicolumn{2}{c|}{} & Sampling Rate vs. Speed. \\
\hline \multirow{2}{*}{$\begin{array}{c}\text { Sampling } \\
\text { Rate }\end{array}$} & \multicolumn{2}{|c|}{$\begin{array}{c}\text { Maximum speed of the } \\
\text { Vehicle to obtain }\end{array}$} \\
\cline { 2 - 3 } & $\begin{array}{c}\text { One measure } \\
\text { In the } 1^{\text {st }} \text { axel }\end{array}$ & $\begin{array}{c}\text { Two measures } \\
\text { In the } 1^{\text {st }} \text { axel }\end{array}$ \\
\hline $2000 \mathrm{~Hz}$ & $720 \mathrm{~km} / \mathrm{h}$ & $360 \mathrm{~km} / \mathrm{h}$ \\
\hline $1000 \mathrm{~Hz}$ & $360 \mathrm{~km} / \mathrm{h}$ & $180 \mathrm{~km} / \mathrm{h}$ \\
\hline $667 \mathrm{~Hz}$ & $240 \mathrm{~km} / \mathrm{h}$ & $120 \mathrm{~km} / \mathrm{h}$ \\
\hline $500 \mathrm{~Hz}$ & $180 \mathrm{~km} / \mathrm{h}$ & $90 \mathrm{~km} / \mathrm{h}$ \\
\hline $400 \mathrm{~Hz}$ & $144 \mathrm{~km} / \mathrm{h}$ & $72 \mathrm{~km} / \mathrm{h}$ \\
\hline $333 \mathrm{~Hz}$ & $120 \mathrm{~km} / \mathrm{h}$ & $60 \mathrm{~km} / \mathrm{h}$ \\
\hline $250 \mathrm{~Hz}$ & $90 \mathrm{~km} / \mathrm{h}$ & $45 \mathrm{~km} / \mathrm{h}$ \\
\hline $167 \mathrm{~Hz}$ & $60 \mathrm{~km} / \mathrm{h}$ & $30 \mathrm{~km} / \mathrm{h}$ \\
\hline $125 \mathrm{~Hz}$ & $45 \mathrm{~km} / \mathrm{h}$ & $22.5 \mathrm{~km} / \mathrm{h}$ \\
\hline $100 \mathrm{~Hz}$ & $36 \mathrm{~km} / \mathrm{h}$ & $18 \mathrm{~km} / \mathrm{h}$ \\
\hline
\end{tabular}

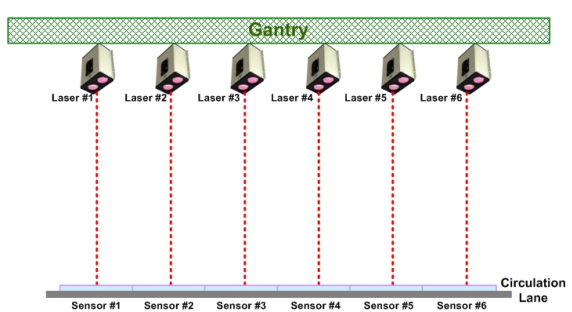

Fig. 5. AVDC - MLFF possible configuration for two circulation lanes.

\section{Experimental Results with the AVDC}

The AVDC considers that a vehicle is present in the system whenever the mean of the collected measures is above a pre determined level. This simple criterion is used to mark the start and end of the ULS data collection and to distinguish which are the data samples above the axels. A schematic of the implemented AVDC is presented in Figure 6 where the laser is collecting measures in a continuous mode when the collected measures are above the referred pre defined level; it is considerate that a vehicle is present in the classification area. When the detection is made the system starts to save all collected measures as well as the information of the treadle/axels. When the mean of the collected measures get smaller than the level it is considered that the vehicle is out of the classification all the information is saved into a file and a picture of the vehicle is taken for better understanding the vehicle type and its characteristics, the system returns to stand by after. The ULS was configured to collect 1000 
measures per second. This value was obtained after several tests and the chosen value seems to be a good trade-off between speed and precision.

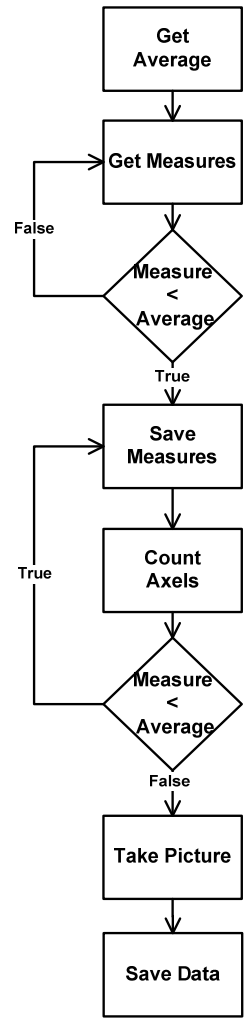

Fig. 6. The AVDC system.

The developed AVDC is already being tested on a Brisa highway toll plaza. The images showed in Figure 7 represent several profiles as well as the images of the corresponding vehicles.

It is possible to observe that the profiles have a high quality and the axels are correctly identified. These results suggest that the system can perform the classification of the vehicle either based on the Portuguese legislation or based on volumetric features derived from the $2 \mathrm{D}$ profile.

\section{ClassificAtion BASED ON PROFILE FEATURES}

\section{A. Eigenprofiles}

The developed classification system uses a method based on PCA. This technique is used as a general feature extraction method in many pattern recognition systems [6]. PCA is used to reduce the dimensionality of datasets which in general simplifies the design of the classifier. This method involves the calculation of the eigenvalue / eigenvectors of the data covariance matrix or a singular value decomposition of a data matrix (usually after mean centering the data for each attribute). This is a data driven method which chooses the better linear projection, in the sense that it maximizes the scatter of all the projected samples.
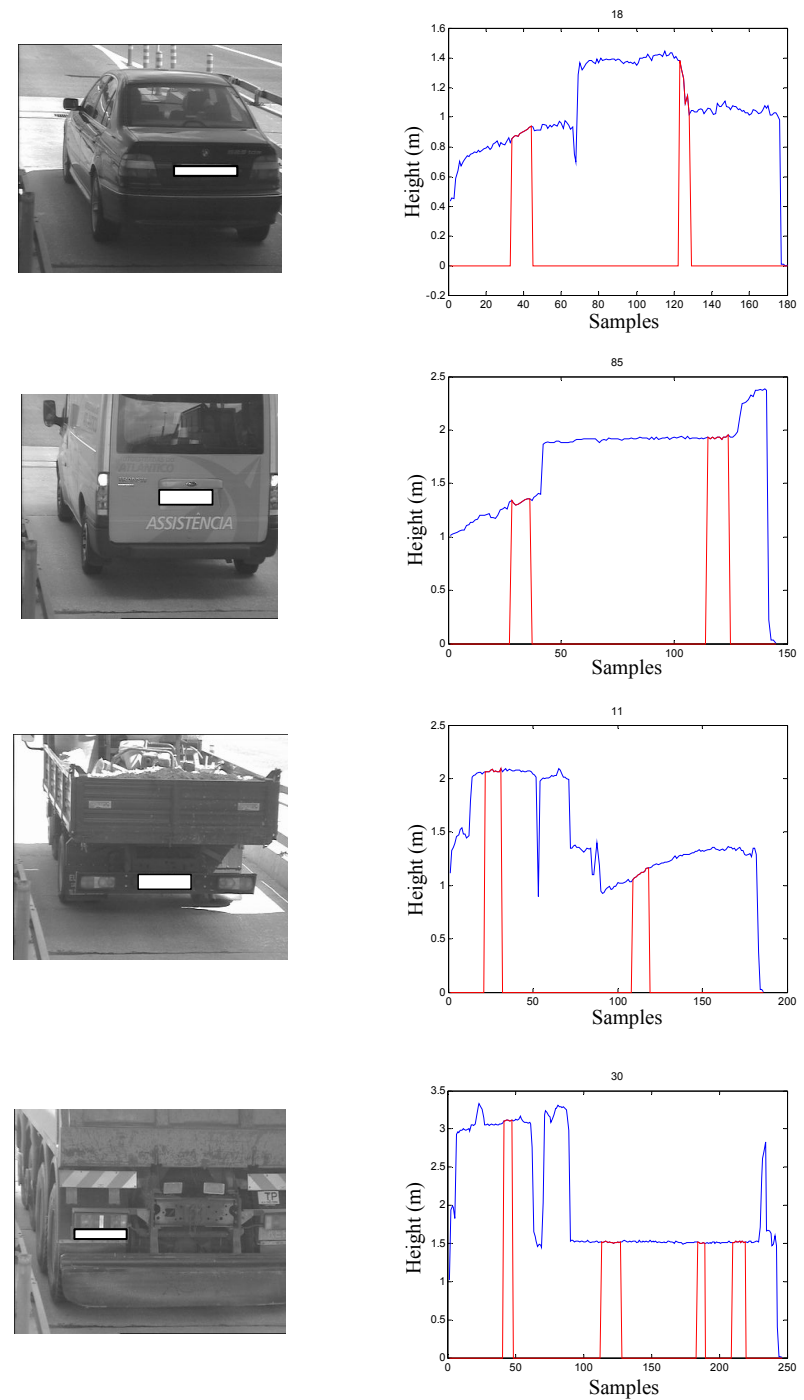

Fig. 7. Obtained Profiles and respective images.

A scheme of the volumetric classification system is presented in Figure 8.

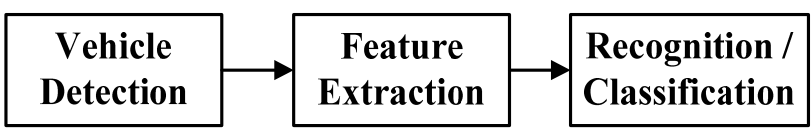

Fig. 8 - Scheme of the volumetric classification system.

Consider a set of $\mathrm{N}$ sample profiles $\left\{x_{1}, x_{2}, \ldots \ldots, x_{N}\right\}$, taking values in an n-dimensional space, and consider that each profile belongs to one of c-classes $\left\{X_{1}, X_{2}, \ldots \ldots, X_{C}\right\}$. Now, a linear transformation can map the original ndimensional profiles into an m-dimensional feature space, with lower dimension than the original. The feature vectors are computed using 


$$
Y_{k}=W^{T} x_{k}
$$

which corresponds to a linear transformation of the raw data. In order to find the matrix $W$, the squared-error criterion

$$
J_{m}=\sum_{k=1}^{N}\left\|x_{k}-\hat{x}_{k}\right\|^{2}=-W^{T} S W+\sum_{k=1}^{N}\left\|x_{k}-\mu\right\|^{2}
$$

has to be minimized which implies the maximization of $W^{T} S W$, where $\mathrm{S}$ is the scatter matrix and $\mu$ the mean profile (which represents the "average" vehicle)

$$
\begin{gathered}
\mu=\frac{1}{N} \sum_{k=1}^{N} x_{k}(3) \\
S_{T}=\sum_{k=1}^{N}\left(x_{K}-\mu\right)\left(x_{K}-\mu\right)^{T}(4)
\end{gathered}
$$

\section{B. Experimental Results with the EigenProfiles}

Several tests were performed to validate the Eigenprofiles. Fig. 9 shows the mean profile obtained using a set of 1059 profiles. These profiles are organized in different classes defined in order of the profile, with different number of profiles within each class. The classes are: Big Truck, Big Van, Car, Jeep, MPV, Open Truck, Pick Up, Small Truck with Box and Small Van.

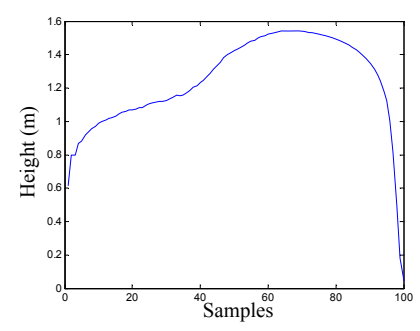

Fig. 9. Mean profile.

Using 120 vehicles for testing the developed system was possible to obtain results like the ones presented in table III. These results were obtained using the nearest neighbor search. For all vehicles the classification had error rate of $6,67 \%$, but if we reduce the number of classes, joining classes with similar profiles, like the MPV and the Jeep, it would reduce the error rate, for this example the error reduced to $2,5 \%$.

\section{Future DeVELOPMENT}

\section{A. Additional Testing of the AVDC}

More testing is needed to make the AVDC a good and reliable tool for classification according to the Portuguese classification, as to the classification based on profile.
TABLE III

Examples of searches.

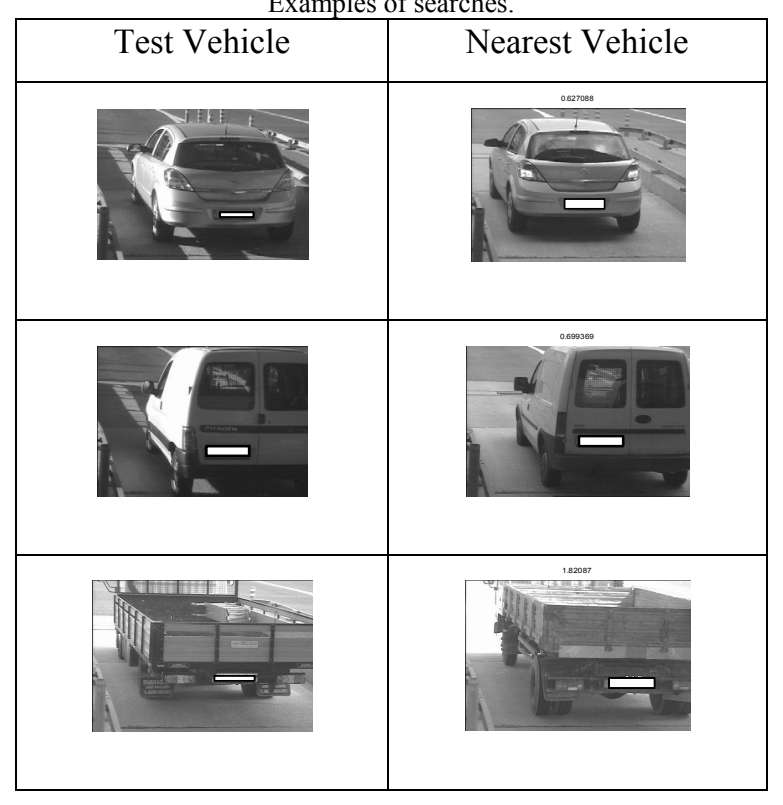

For classification based on profile, it should be developed and tested new feature extraction and classification besides the Eigen profiles. Fisher profiles based on the Multiple Discrete Analysis (MDA) and other classification and decision techniques are also being discussed to future improvements of the AVDC profile based classification.

\section{B. Setup of the $M L F F$}

The setup of the MLFF is still under development. Although there is already some clues of how the setup should be, like and number of lasers per lane, it still needs some development and several testing.

\section{CONCLUSIONS}

In this paper we have presented a vehicle detection and classification system. The system adopts a range laser sensor to perform detection and also to obtain the profile of each vehicle. The system can evaluate the height at the first axel and the number of axels. This is all the information needed to classify the vehicle according to Portuguese law. Despite the fact that $2 \mathrm{D}$ profile is not an essential feature for performing the classification under the actual Portuguese situation, it is an unquestionable benefit of the proposed system, namely if we are considering the fast changes that are occurring in motorways toll collection environments (MLFF). For this purpose, Eigenprofiles were evaluated. Using the Eigenprofiles coefficients a nearest neighbor classifier algorithm was used and its accuracy evaluated. The results obtained so far are very promising for future work where other feature extraction and classifying systems will be put in use for comparison purposes. 


\section{ACKNOWLEDGMENT}

The development of this system is being made in cooperation with a Portuguese motorway operator, Brisa, being this way possible to test the system in real situations. The authors would like to thank to Bruno Basílio, Gastão Jacquet and Miguel Machado from Brisa.

\section{REFERENCES}

[1] Laser Technology Inc. - www.lasertech.com

[2] Efkon - www.efkon.com

[3] Kapsch - www.kapsch.at

[4] Hussain, K. F. and Moussa, G. S. "Laser Intensity Vehicle

Classification System Based on Random Neural Network", ACM Southeast Regional Conference, 2005

[5] Totha, C. K., Barsib, A. and Lovasb, T. - "Vehicle Recognition From LIDAR Data", International Society for Photogrammetry and Remote

Sensing

[6] Belhumeur, P.N., Hespanha, J.P and Kriegman, D.J. - "Eigenfaces vs.

Fisherfaces: recognition using class specific linear projection", IEEE

Transactions on Analysis and Machine Intelligence, Volume 19, Issue 7, Jul 1997

[7] TDS - Quick Change Fiber Optic Treadle

[8] Duda, R.O., Hart, P.E. and Stork, D.G. - "Pattern Classification", 2nd

Edition

[9] Hiroyuki Kuwagaki - "Vehicle Classification Using Profile", US Patent 5392034

[10] Petrovi'c, V. S. and Cootes, T. F. - "Analysis of Features for Rigid Structure Vehicle Type Recognition", Imaging Science Bimedical Engineering, University of Manchester, UK

[11] Jolly, Marie-Pierre D., Lakshmanan, Sridhar and Jain, Anil K. -

"Vehicle Segmentation and Classification Using Deformable Templates", IEEE Transactions on Pattern Analysis and Machine Intelligence, Mar

1996

[12] Gupte, Surendra, Masoud, Osama, Martin R. F. K., and Papanikolopoulos, N. P. - "Detection and Classification of Vehicles", IEEE Transactions on Intelligent Transportation Systems, Volume 3, Issue 1, Mar 2002

[13] Ma, Xiaoxu, Grimson, W. Eric L.- "Edge-based rich representation for vehicle classification", Tenth IEEE International Conference on Computer Vision, Oct 2005 